\title{
Microsatellites in Different Eukaryotic Genomes: Survey and Analysis
}

\author{
Gábor Tóth, ${ }^{1,3}$ Zoltán Gáspári, ${ }^{1}$ and Jerzy Jurka ${ }^{2}$ \\ ${ }^{1}$ Department of Genetics, Eötvös Loránd University, Budapest, Hungary; ${ }^{2}$ Genetic Information Research Institute, Sunnyvale, \\ California 94089 USA
}

\begin{abstract}
We examined the abundance of microsatellites with repeated unit lengths of 1-6 base pairs in several eukaryotic taxonomic groups: primates, rodents, other mammals, nonmammalian vertebrates, arthropods, Caenorhabditis elegans, plants, yeast, and other fungi. Distribution of simple sequence repeats was compared between exons, introns, and intergenic regions. Tri- and hexanucleotide repeats prevail in protein-coding exons of all taxa, whereas the dependence of repeat abundance on the length of the repeated unit shows a very different pattern as well as taxon-specific variation in intergenic regions and introns. Although it is known that coding and noncoding regions differ significantly in their microsatellite distribution, in addition we could demonstrate characteristic differences between intergenic regions and introns. We observed striking relative abundance of $(C C G)_{n} \bullet(C G G)_{n}$ trinucleotide repeats in intergenic regions of all vertebrates, in contrast to the almost complete lack of this motif from introns. Taxon-specific variation could also be detected in the frequency distributions of simple sequence motifs. Our results suggest that strand-slippage theories alone are insufficient to explain microsatellite distribution in the genome as a whole. Other possible factors contributing to the observed divergence are discussed.
\end{abstract}

Microsatellites or simple sequence repeats (SSRs) are tandemly repeated tracts of DNA composed of 1-6 base pair (bp) long units. They are ubiquitous in prokaryotes and eukaryotes, present even in the smallest bacterial genomes (Field and Wills 1996; Hancock 1996a). A subset of SSRs, namely trinucleotide repeats, are of great interest because of the role they play in many human neurodegenerative disorders (fragile X syndrome, Huntington's disease, myotonic dystrophy, spinal-bulbar muscular atrophy, spinocerebellar ataxia, etc.; for reviews, see Warren and Nelson 1993; Bates and Lehrach 1994; Reddy and Housman 1997) and in some human cancers, e.g. hereditary nonpolyposis colorectal carcinoma (Wooster et al. 1994; Arzimanoglou et al. 1998). The alteration responsible for these genetic diseases is the expansion of triplet repeats, where the rate of mutation depends on the number of tandem units within the repeat. Hence the term 'dynamic mutation' was coined by Richards and Sutherland (1992).

Microsatellites can be found anywhere in the genome, both in protein-coding and noncoding regions. Because of their high mutability, microsatellites are thought to play a significant role in genome evolution by creating and maintaining quantitative genetic variation (Tautz et al. 1986; Kashi et al. 1997). In promoter regions, the length of SSRs may influence transcriptional activity (Kashi et al. 1997). Length of polyglutamine or polyproline tracts encoded by SSRs may

\footnotetext{
${ }^{3}$ Corresponding author.
}

E-MAIL tothg@ludens.elte.hu; FAX (+36-1) 266-2694. affect protein-protein interactions involving transcription factors (Gerber et al. 1994; Perutz et al. 1994).

It has been shown that SSRs in exons are less abundant than in noncoding regions (Hancock 1995), and that different taxa exhibit different preferences for SSR types (Beckmann and Weber 1992; Lagercrantz et al. 1993; Tautz and Schlötterer 1994). Moreover, the overall microsatellite content in the genome correlates with the genome size of the organisms (Hancock 1996b).

SSRs are inherently unstable. Two models have been proposed to explain microsatellite generation and instability: DNA polymerase slippage and unequal recombination. The first model involves transient dissociation of the replicating DNA strands, followed by misaligned reassociation (Richards and Sutherland 1994). The slipped structure may be stabilized by hairpin, triplex, or quadruplex arrangement of DNA strands (for review, see Pearson and Sinden 1998; Sinden 1999). Thus, it is expected that those repeats that are able to form such alternative DNA conformations would be generated more frequently than others. The possible structures of triplet repeats involved in human diseases have been studied extensively. The repeats that show a considerable potential to form alternative structures include $(\mathrm{CTG})_{\mathrm{n}} \bullet(\mathrm{CAG})_{\mathrm{n}},(\mathrm{CCG})_{\mathrm{n}} \bullet(\mathrm{CGG})_{\mathrm{n}}$, $(\mathrm{GAA})_{\mathrm{n}} \bullet(\mathrm{TTC})_{\mathrm{n}},(\mathrm{AGG})_{\mathrm{n}} \bullet(\mathrm{CCT})_{\mathrm{n}}$, and $(\mathrm{TGG})_{\mathrm{n}} \bullet(\mathrm{CCA})_{\mathrm{n}}$ (Gacy et al. 1995; Bidichandani et al. 1998; Usdin 1998). However, some sequences with theoretically high hairpin-forming potential [e.g. (CCG) ${ }_{n}$ ] show the slowest in vitro slippage rate (Schlötterer and Tautz 1992). Moreover, the rate of alterations is likely to be 
controlled at multiple steps in vivo. An active role of the DNA mismatch repair system to stabilize simple sequence repeats has been revealed in Escherichia coli, yeast, and humans (for review, see Sia et al. 1997). Although a number of experimental results argue in favor of the above model, homologous recombination may also result in genetic instability of certain SSRs (Jakupciak and Wells 1999).

We can expect that the fixation of de novogenerated SSRs is determined by the interplay of several factors, of which the repeat type, the genomic position of the SSR, and the genetic-biochemical background of the cell are the most important. In our study we addressed the questions of whether the abundance of various microsatellite types is similar or not in different taxonomic groups and how SSR frequencies differ in exons, introns, and intergenic regions. We intended to give a detailed picture analyzing all possible (501) SSR motifs to complement the results of a previous study on primate DNA sequence data (Jurka and Pethiyagoda 1995), and place them into comparative evolutionary perspective.

\section{RESULTS}

We examined the distribution of perfect SSRs over 12bp long, so if not explicitly stated otherwise, our results described here apply to microsatellites meeting this criterion. To assess expandability of the repeats, we also analyzed perfect repeats longer than $24 \mathrm{bp}$ (see Methods) and compared the results to those obtained using the shorter cutoff length. Data presented below always refer to duplex DNA, even if we show only the sequence of the repeated motif on one strand for simplicity, i.e. notations like $\mathrm{AC}$ and $(\mathrm{AC})_{\mathrm{n}} \bullet(\mathrm{GT})_{\mathrm{n}}$ are equivalent.

The nonoverlapping groups of DNA sequences used in this study will be referred to as taxonomic groups or taxa. These groups represent either individual species (Caenorhabditis elegans and Saccharomyces cerevisiae), or groups of related species such as Primates, Rodentia, and Mammalia. Thus our taxa are defined rather arbitrarily based primarily on sequence availability (see Methods). We carried out the analyses on sequences classified into three genomic regions (intergenic regions, introns, and exons), and on a superset referred to as all sequences. The latter contained all sequence entries that passed the filtering criteria described in Methods, even if they could not be assigned to genomic regions.

To estimate database bias caused by the use of GenBank, we also included the full sequence of the human chromosome 22 in our study. The results obtained for chromosome 22 are in good agreement with those for all primate sequences, confirming the validity of our approach. The $30 \%$ increase in total micro- satellite content in the full chromosomal sequence (see the last column of Table 1 ) is mostly due to greater abundance of $(\mathrm{A}+\mathrm{T})$-rich repeats, especially poly $(\mathrm{A} / \mathrm{T})$ tracts (Tables 2 and 6 ).

To assess the contribution of repeated unit length to microsatellite abundance, we calculated the total lengths of all mono-, di-, tri-, tetra-, penta-, and hexanucleotide repeats per megabase pair $(\mathrm{Mbp})$ of DNA sequence (Table 1). In exons, trinucleotide repeats are invariably the most abundant in all taxa, with hexanucleotide repeats being the second most common. Intergenic regions and introns, however, contain more hexanucleotide repeats than exons do, Embryophyta and $S$. cerevisiae introns being the only exceptions to this rule.

In primates, mononucleotide repeats are the most copious. In introns and intergenic regions they are more than twice as frequent as di- and tetranucleotide repeats. The latter are of similar abundance, and interestingly, much more frequent than trinucleotide repeats. In rodents, repeats with dinucleotide units are about three times more frequent than those with mononucleotides. Dinucleotide repeats are dominant in introns and intergenic regions of many other taxa, except for Primates, Embryophyta, S. cerevisiae, and Fungi. In rodent introns and intergenic regions, the rarity of triplet repeats is also quite pronounced in comparison to di- and tetranucleotide repeats.

The relative abundance of tetranucleotide over trinucleotide repeats in introns and intergenic regions is characteristic of all vertebrate taxa but not of any other taxonomic group studied. In all mammalian taxa, even pentanucleotide repeats are more frequent in introns and intergenic regions than triplet repeats. In invertebrates and fungi, tetranucleotide repeats constitute the less frequent class of microsatellite in introns and intergenic regions, whereas in vascular plants they are comparably rare as hexanucleotide repeats.

When comparing various taxonomic groups, it is evident that rodents adopt much more microsatellites than any other group we examined. C. elegans, however, contains the least SSRs per one Mbp of DNA, less than $S$. cerevisiae and other fungi.

A more detailed picture could be drawn when we analyzed the distribution of SSRs by the sequence of the repeated motif. Results obtained for mono-, di-, and trinucleotide repeats are shown in Tables 2-5. The most frequent tetra-, penta-, and hexanucleotide repeats are listed in Tables 6-9. More data are available online in our SSRDB database at http://genetics. elte.hu/ssr.

\section{Mononucleotide Repeats}

In general, poly $(\mathrm{A} / \mathrm{T})$ tracts are more abundant in each taxon than poly(C/G) sequences (Tables 2-5). This difference is the least characteristic in C. elegans and most 
Table 1. Total Lengths ${ }^{a}$ of Simple Sequence Repeats by Repeated Unit Length

\begin{tabular}{|c|c|c|c|c|c|c|c|c|}
\hline \multirow[b]{2}{*}{ Taxonomic group } & \multirow{2}{*}{$\begin{array}{c}\text { Genomic } \\
\text { region }\end{array}$} & \multicolumn{6}{|c|}{ Length of repeated motif (bp) } & \multirow[b]{2}{*}{ Total } \\
\hline & & 1 & 2 & 3 & 4 & 5 & 6 & \\
\hline \multicolumn{9}{|l|}{ Primates } \\
\hline & $\begin{array}{l}\text { all } \\
\text { intergenic }\end{array}$ & 3429 & 1643 & 477 & 1368 & 898 & 341 & 8156 \\
\hline & regions & 3880 & 1709 & 517 & 1464 & 991 & 385 & 8946 \\
\hline & introns & 4137 & 1506 & 424 & 1428 & 988 & 392 & 8875 \\
\hline & exons & 49 & 10 & 1126 & 29 & 57 & 244 & 1515 \\
\hline \multicolumn{9}{|l|}{$\begin{array}{l}\text { Human } \\
\text { chromosome } 22\end{array}$} \\
\hline \multirow{2}{*}{\multicolumn{9}{|c|}{ Rodentia }} \\
\hline & & & & & & & & \\
\hline & $\begin{array}{l}\text { all } \\
\text { intergenic }\end{array}$ & 1839 & 5461 & 1196 & 2942 & 1417 & 1034 & 13889 \\
\hline & regions & 2192 & 5928 & 1230 & 2823 & 1577 & 740 & 14490 \\
\hline & introns & 2182 & 5837 & 1123 & 3009 & 1399 & 922 & 14472 \\
\hline & exons & 62 & 70 & 1557 & 63 & 116 & 620 & 2488 \\
\hline \multicolumn{9}{|l|}{ Mammalia } \\
\hline & $\begin{array}{l}\text { all } \\
\text { intergenic }\end{array}$ & 1397 & 2312 & 532 & 915 & 774 & 693 & 6623 \\
\hline & regions & 1954 & 4666 & 531 & 1529 & 1115 & 1155 & 10950 \\
\hline & introns & 1967 & 2202 & 395 & 792 & 685 & 637 & 6678 \\
\hline & exons & 69 & 88 & 876 & 19 & 18 & 356 & 1426 \\
\hline \multicolumn{9}{|l|}{ Vertebrata } \\
\hline & $\begin{array}{l}\text { all } \\
\text { intergenic }\end{array}$ & 1418 & 2449 & 1069 & 1219 & 109 & $\angle \angle O$ & 1144 \\
\hline & regions & 2193 & 3363 & 1127 & 1766 & 1201 & 320 & 9970 \\
\hline & introns & 1476 & 3193 & 861 & 1502 & 585 & 142 & 7759 \\
\hline & exons & 49 & 0 & 823 & 0 & 26 & 75 & 973 \\
\hline \multicolumn{9}{|l|}{ Arthropoda } \\
\hline & $\begin{array}{l}\text { all } \\
\text { intergenic }\end{array}$ & 985 & 1403 & 956 & 439 & 732 & 875 & 5390 \\
\hline & regions & 1462 & 2259 & 1128 & 621 & 1110 & 1090 & 7670 \\
\hline & introns & 950 & 1627 & 728 & 461 & 735 & 917 & 5418 \\
\hline & exons & 12 & 34 & 1566 & 0 & 21 & 591 & 2224 \\
\hline \multicolumn{9}{|l|}{ C. elegans } \\
\hline & $\begin{array}{l}\text { all } \\
\text { intergenic }\end{array}$ & 428 & 556 & 337 & 144 & 225 & 449 & 2139 \\
\hline & regions & 573 & 822 & 414 & 198 & 310 & 574 & 2891 \\
\hline & introns & 512 & 549 & 228 & 169 & 283 & 556 & 2297 \\
\hline & exons & 43 & 54 & 308 & 18 & 38 & 116 & 577 \\
\hline \multicolumn{7}{|l|}{ Embryophyta } & & \\
\hline & intergenic & 1243 & 1008 & 000 & 104 & 491 & $2 / 2$ & 4139 \\
\hline & regions & 2012 & 1715 & 869 & 303 & 781 & 334 & 6014 \\
\hline & introns & 1380 & 1322 & 576 & 260 & 547 & 207 & 4292 \\
\hline & exons & 18 & 50 & 1119 & 2 & 29 & 303 & 1521 \\
\hline \multicolumn{9}{|l|}{ S. cerevisiae } \\
\hline & $\begin{array}{l}\text { all } \\
\text { intergenic }\end{array}$ & 1075 & 580 & 646 & 93 & 204 & 406 & 3004 \\
\hline & regions & 3140 & 1875 & 512 & 273 & 494 & 532 & 6826 \\
\hline & introns & 3012 & 1437 & 516 & 162 & 509 & 288 & 5924 \\
\hline & exons & 36 & 19 & 706 & 7 & 52 & 330 & 1150 \\
\hline \multicolumn{9}{|l|}{ Fungi } \\
\hline & $\begin{array}{l}\text { all } \\
\text { intergenic }\end{array}$ & 905 & 272 & 485 & 194 & 395 & 426 & 2677 \\
\hline & regions & 2080 & 555 & 550 & 421 & 925 & 548 & 5079 \\
\hline & introns & 2075 & 1013 & 951 & 458 & 659 & 661 & 5817 \\
\hline & exons & 9 & 4 & 381 & 2 & 35 & 219 & 650 \\
\hline
\end{tabular}

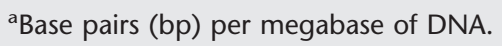

pronounced in primates. The total length of mononucleotide repeats, taking together both patterns, is also greatest in primates (Table 1). Nonmammalian vertebrates show the second highest ratio of poly $(\mathrm{C} / \mathrm{G})$ 
Tóth et al.

Table 2. Total Lengths of Mono-, Di-, and Trinucleotide Repeats in All Sequences ${ }^{\mathrm{a}}$

\begin{tabular}{|c|c|c|c|c|c|c|c|c|c|c|}
\hline \multirow[b]{2}{*}{$\begin{array}{l}\text { Repeated } \\
\text { unit }\end{array}$} & \multicolumn{10}{|c|}{ Taxonomic group } \\
\hline & primates & $\begin{array}{l}\text { human } \\
\text { chr } 22^{b}\end{array}$ & rodentia & mammalia & vertebrata & arthropoda & $\begin{array}{c}\text { C. } \\
\text { elegans }\end{array}$ & embryophyta & $\begin{array}{c}\text { S. } \\
\text { cerevisiae }\end{array}$ & fungi \\
\hline A & 3418 & 5126 & 1634 & 1291 & 1051 & 875 & 227 & 1221 & 1069 & 872 \\
\hline C & 11 & 15 & 205 & 106 & 367 & 110 & 201 & 24 & 6 & 33 \\
\hline AC & 1033 & 981 & 3468 & 1333 & 1496 & 825 & 163 & 85 & 67 & 81 \\
\hline AG & 293 & 261 & 1619 & 854 & 334 & 235 & 222 & 333 & 19 & 58 \\
\hline AT & 314 & 266 & 354 & 109 & 616 & 340 & 170 & 648 & 494 & 133 \\
\hline CG & 3 & 3 & 20 & 16 & 3 & 3 & 1 & 1 & - & - \\
\hline AAC & 110 & 128 & 149 & 49 & 66 & 189 & 19 & 139 & 125 & 108 \\
\hline AAG & 42 & 55 & 217 & 19 & 36 & 17 & 105 & 332 & 119 & 59 \\
\hline AAT & 161 & 211 & 122 & 30 & 350 & 100 & 70 & 108 & 176 & 119 \\
\hline ACC & 25 & 56 & 136 & 17 & 54 & 58 & 28 & 42 & 6 & 36 \\
\hline ACG & 0 & 1 & 1 & 15 & 3 & 26 & 12 & 11 & 28 & 12 \\
\hline ACT & 6 & 2 & 8 & 4 & 21 & 16 & 11 & 15 & 17 & 15 \\
\hline AGC & 32 & 32 & 222 & 201 & 166 & 387 & 23 & 38 & 80 & 55 \\
\hline AGG & 42 & 60 & 226 & 97 & 182 & 58 & 15 & 52 & 13 & 31 \\
\hline ATC & 26 & 35 & 34 & 4 & 93 & 86 & 48 & 119 & 82 & 32 \\
\hline CCG & 33 & 24 & 81 & 96 & 98 & 19 & 6 & 24 & - & 18 \\
\hline
\end{tabular}

${ }^{a}$ Base pairs (bp) per megabase of DNA.

bHuman chromosome 22 sequence.

to poly(A/T). Besides C. elegans, they constitute the only group where poly $(\mathrm{C} / \mathrm{G})$ repeats appear in exons in a proportion comparable to poly(A/T) (Table 5). Intergenic regions show an interesting preference for poly $(\mathrm{C} / \mathrm{G})$ over poly $(\mathrm{A} / \mathrm{T})$ in C. elegans (Table 3$)$. Introns contain more poly $(\mathrm{A} / \mathrm{T})$ than $\operatorname{poly}(\mathrm{C} / \mathrm{G})$ repeats in each taxon (Table 4).
Dinucleotide Repeats

Dinucleotide repeats are most abundant in rodents and the least frequent in fungi (Table 1). Characteristic differences between taxa can only be observed for intergenic regions and introns (Tables 3 and 4) because of the rarity of dinucleotide repeats in exons (Table 5). Curiously, we have found one 16-bp long CG repeat in

Table 3. Total Lengths of Mono-, Di-, and Trinucleotide Repeats in Intergenic Regions ${ }^{\mathrm{a}}$

\begin{tabular}{|c|c|c|c|c|c|c|c|c|c|}
\hline \multirow[b]{2}{*}{$\begin{array}{l}\text { Repeated } \\
\text { unit }\end{array}$} & \multicolumn{9}{|c|}{ Taxonomic group } \\
\hline & primates & rodentia & mammalia & vertebrata & arthropoda & $\begin{array}{c}\text { C. } \\
\text { elegans }\end{array}$ & embryophyta & $\begin{array}{c}\text { S. } \\
\text { cerevisiae }\end{array}$ & fungi \\
\hline A & 3864 & 1956 & 1868 & 1612 & 1333 & 252 & 1975 & 3121 & 2024 \\
\hline C & 16 & 236 & 86 & 581 & 129 & 321 & 37 & 19 & 56 \\
\hline$A C$ & 1049 & 3733 & 2654 & 2070 & 1159 & 249 & 115 & 266 & 140 \\
\hline AG & 327 & 1799 & 1766 & 294 & 437 & 353 & 521 & 57 & 136 \\
\hline AT & 329 & 363 & 246 & 999 & 663 & 219 & 1077 & 1552 & 279 \\
\hline CG & 4 & 33 & - & - & - & 1 & 2 & - & - \\
\hline AAC & 115 & 156 & 28 & 83 & 437 & 16 & 106 & 23 & 104 \\
\hline AAG & 40 & 280 & 31 & 50 & 36 & 143 & 377 & 35 & 84 \\
\hline AAT & 188 & 168 & 77 & 497 & 196 & 98 & 198 & 321 & 162 \\
\hline ACC & 23 & 136 & - & 45 & 47 & 23 & 21 & - & 60 \\
\hline ACG & - & - & - & - & 32 & 14 & 10 & 9 & 6 \\
\hline ACT & 4 & 3 & 13 & 8 & 10 & 16 & 14 & 36 & 30 \\
\hline AGC & 25 & 154 & 236 & 84 & 255 & 18 & 14 & 39 & 19 \\
\hline AGG & 45 & 204 & 56 & 148 & 32 & 14 & 26 & - & 34 \\
\hline ATC & 19 & 41 & - & 112 & 75 & 65 & 88 & 49 & 43 \\
\hline CCG & 58 & 88 & 90 & 100 & 8 & 7 & 15 & - & 8 \\
\hline
\end{tabular}

a Base pairs (bp) per megabase of DNA. 
Table 4. Total Lengths of Mono-, Di-, and Trinucleotide Repeats in Introns ${ }^{\mathrm{a}}$

\begin{tabular}{|c|c|c|c|c|c|c|c|c|c|}
\hline \multirow[b]{2}{*}{$\begin{array}{l}\text { Repeated } \\
\text { unit }\end{array}$} & \multicolumn{9}{|c|}{ Taxonomic group } \\
\hline & primates & rodentia & mammalia & vertebrata & arthropoda & $\begin{array}{c}\text { C. } \\
\text { elegans }\end{array}$ & embryophyta & $\begin{array}{c}\text { S. } \\
\text { cerevisiae }\end{array}$ & fungi \\
\hline A & 4125 & 1893 & 1743 & 1033 & 851 & 335 & 1341 & 2994 & 1868 \\
\hline C & 12 & 289 & 224 & 443 & 99 & 177 & 39 & 18 & 207 \\
\hline AC & 1012 & 3782 & 1348 & 2155 & 749 & 151 & 168 & 26 & 511 \\
\hline AG & 268 & 1741 & 698 & 412 & 469 & 173 & 444 & 26 & 140 \\
\hline AT & 221 & 300 & 124 & 626 & 409 & 224 & 710 & 1385 & 362 \\
\hline CG & 5 & 14 & 32 & - & - & 1 & - & - & - \\
\hline AAC & 114 & 156 & 194 & 105 & 78 & 11 & 121 & - & 145 \\
\hline AAG & 33 & 266 & 20 & 11 & 33 & 70 & 176 & 20 & 34 \\
\hline AAT & 171 & 165 & 24 & 343 & 199 & 67 & 170 & 434 & 537 \\
\hline ACC & 14 & 118 & - & 34 & 51 & 9 & 11 & - & 14 \\
\hline ACG & - & - & - & - & 18 & 6 & - & 39 & - \\
\hline $\mathrm{ACT}$ & 10 & 9 & - & - & 56 & 8 & 16 & 23 & - \\
\hline AGC & 16 & 114 & 117 & 43 & 195 & 16 & 13 & - & 103 \\
\hline AGG & 39 & 253 & 40 & 168 & 11 & 12 & 7 & - & 45 \\
\hline ATC & 26 & 42 & - & 157 & 78 & 26 & 57 & - & 53 \\
\hline CCG & 1 & - & - & - & 9 & 3 & 5 & - & 20 \\
\hline
\end{tabular}

a'Base pairs (bp) per megabase of DNA.

the protein-coding region of beta one adrenergic receptor gene from Canis familiaris. Otherwise, CG repeats are very rare.

In all vertebrates and arthropods, AC is the most frequent dinucleotide repeat motif (Tables 2-4). C. elegans prefers AG in intergenic regions, AT in introns. In embryophytes, yeast, and fungi, AT repeats are the most frequent in general, except for introns in fungi where AC is more abundant (Table 4).

\section{Trinucleotide Repeats}

Trinucleotide repeats can be found in each genomic region with a significant frequency (Tables 2-5). However, the frequency distribution by repeat type shows

Table 5. Total Lengths of Mono-, Di-, and Trinucleotide Repeats in Exons ${ }^{a}$

\begin{tabular}{|c|c|c|c|c|c|c|c|c|c|}
\hline \multirow[b]{2}{*}{$\begin{array}{l}\text { Repeated } \\
\text { unit }\end{array}$} & \multicolumn{9}{|c|}{ Taxonomic group } \\
\hline & primates & rodentia & mammalia & vertebrata & arthropoda & $\begin{array}{c}\text { C. } \\
\text { elegans }\end{array}$ & embryophyta & $\begin{array}{c}\text { S. } \\
\text { cerevisiae }\end{array}$ & fungi \\
\hline A & 49 & 62 & 69 & 30 & 12 & 23 & 17 & 36 & 9 \\
\hline C & - & - & - & 19 & - & 20 & 1 & - & - \\
\hline AC & 4 & 29 & - & - & 21 & 16 & 4 & 5 & 2 \\
\hline AG & 6 & 24 & 69 & - & 6 & 29 & 39 & - & - \\
\hline AT & - & 17 & - & - & 7 & 9 & 7 & 14 & 2 \\
\hline CG & - & - & 19 & - & - & - & - & - & - \\
\hline AAC & 8 & - & - & - & 220 & 33 & 253 & 156 & 107 \\
\hline AAG & 57 & 29 & 22 & 43 & - & 81 & 317 & 147 & 55 \\
\hline AAT & - & - & - & - & 10 & 19 & 4 & 123 & 34 \\
\hline $\mathrm{ACC}$ & 58 & 184 & 22 & 46 & 142 & 65 & 89 & 12 & 26 \\
\hline ACG & 5 & - & 65 & 15 & 21 & 10 & 20 & 27 & 12 \\
\hline ACT & 5 & - & - & - & - & 6 & 13 & 5 & 5 \\
\hline AGC & 381 & 889 & 376 & 337 & 954 & 31 & 108 & 112 & 67 \\
\hline AGG & 192 & 143 & 219 & 210 & 83 & 14 & 106 & 21 & 27 \\
\hline ATC & 26 & 22 & 18 & 15 & 58 & 41 & 138 & 103 & 22 \\
\hline CCG & 394 & 290 & 154 & 157 & 78 & 8 & 71 & - & 26 \\
\hline
\end{tabular}

a Base pairs (bp) per megabase of DNA. 


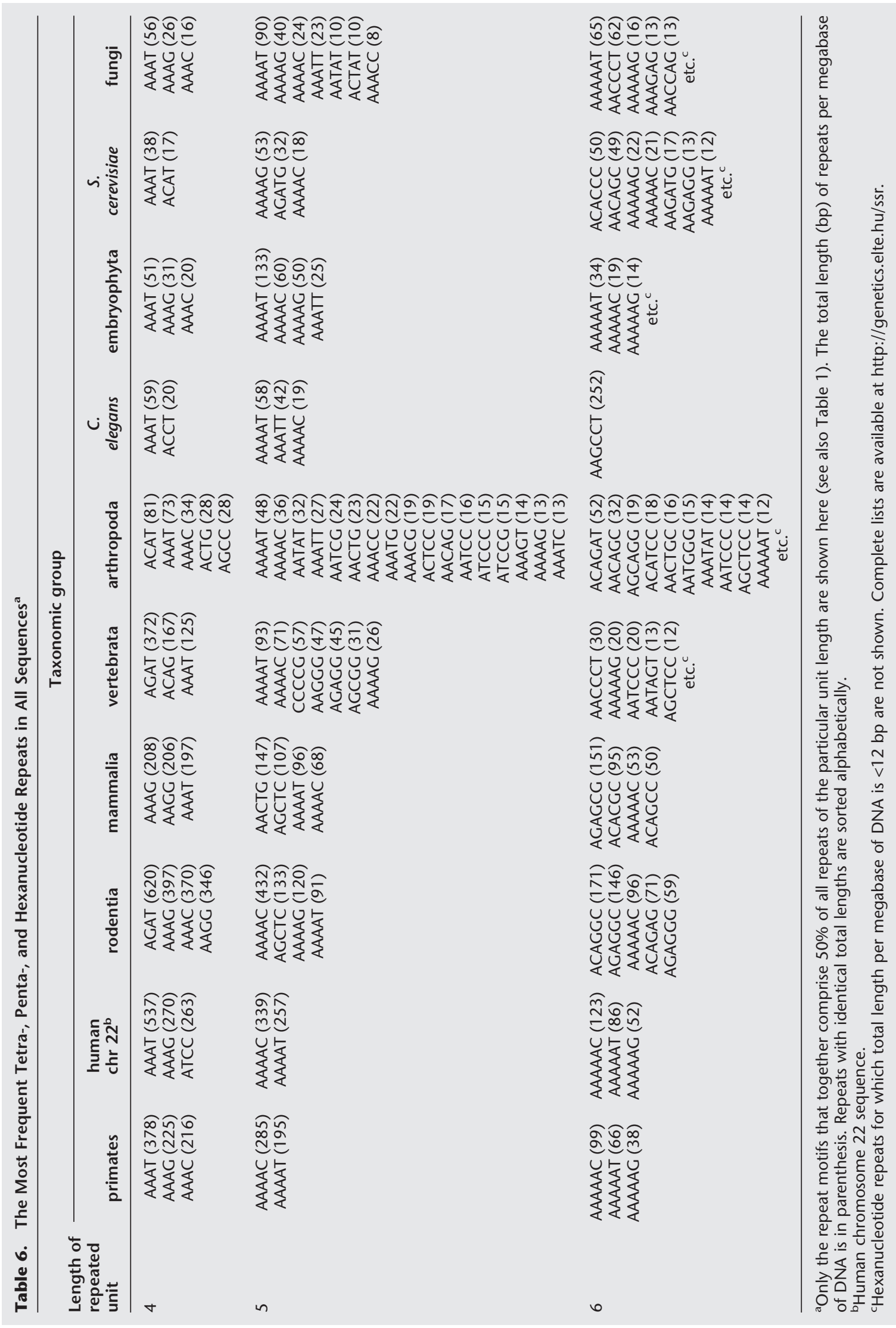




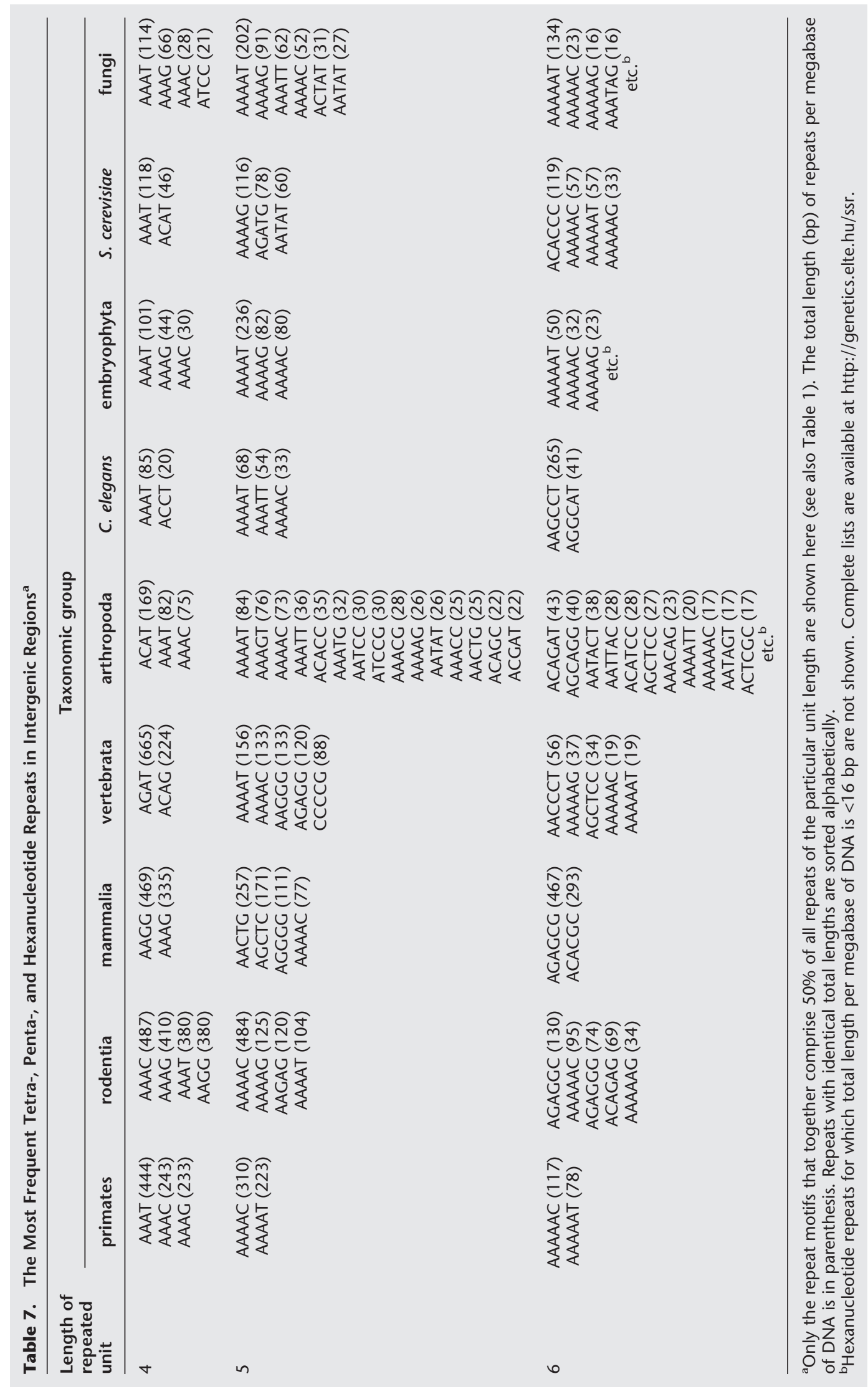




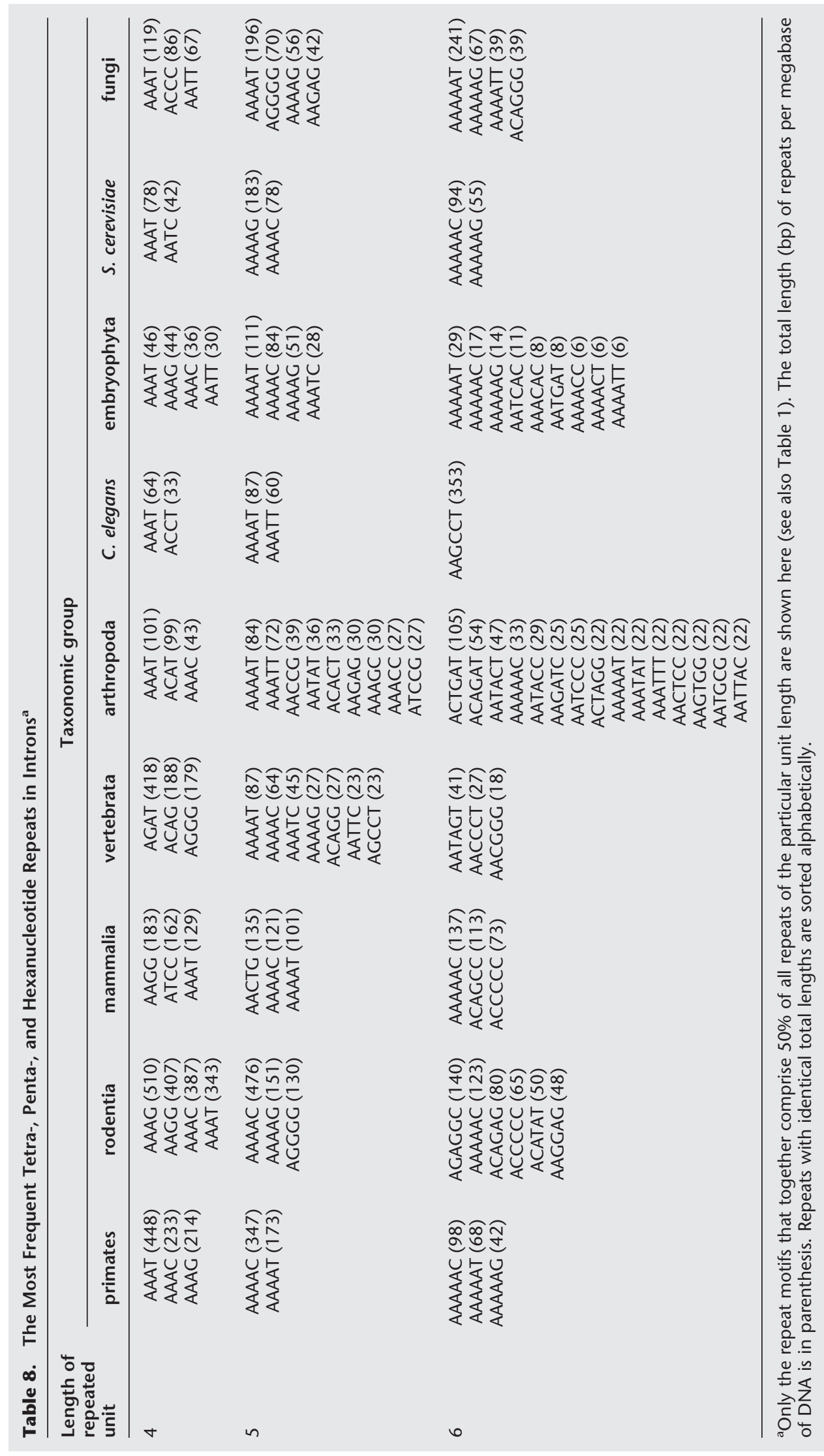




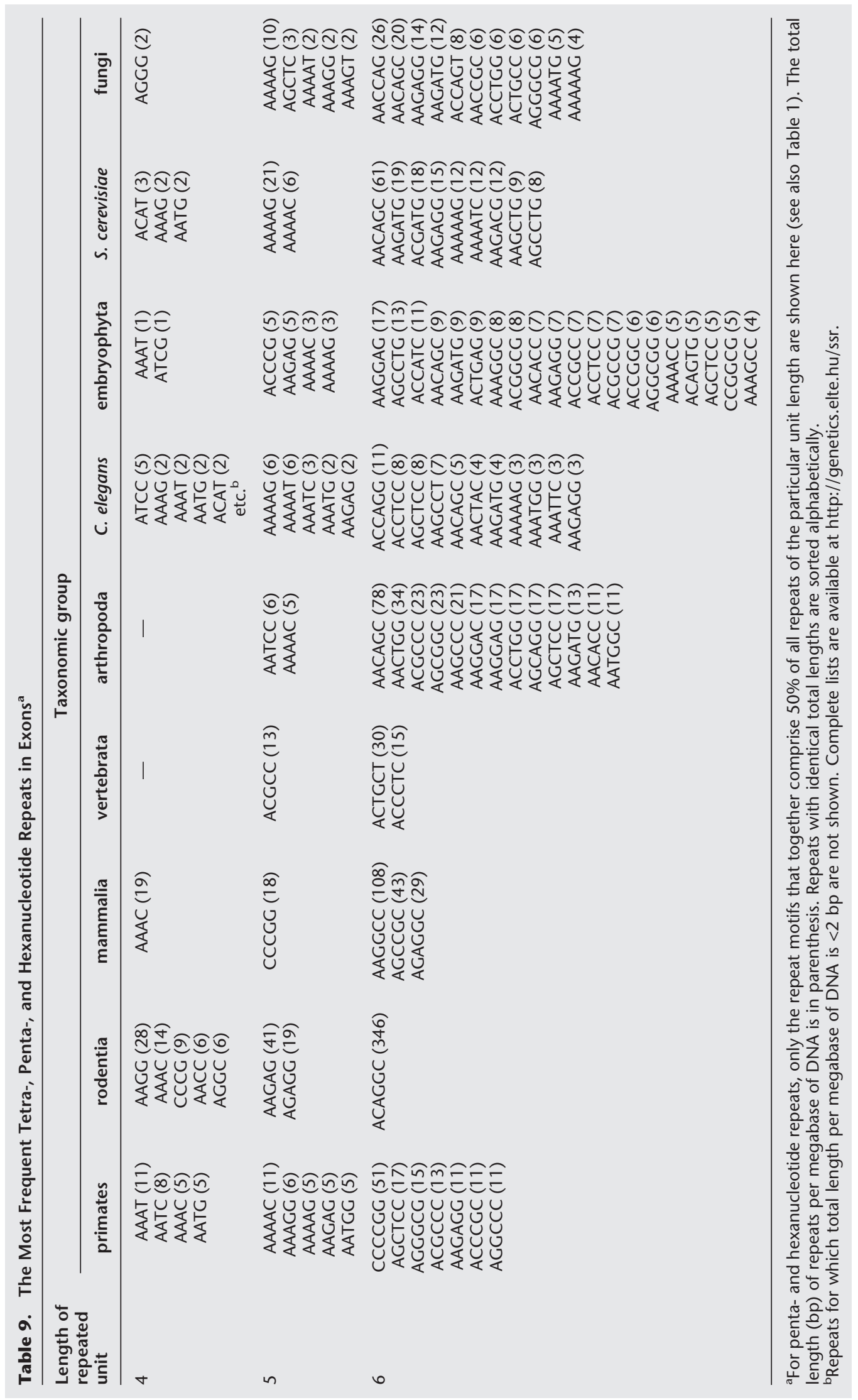


major differences in various genomic regions and among taxa. In all vertebrates, $(\mathrm{G}+\mathrm{C})$-rich repeats dominate in exons, whereas they are less pronounced in other regions. AAC and AAG are the most frequent repeat types in Embryophyta exons and interesting relative abundance of $(\mathrm{A}+\mathrm{T})$-rich repeats can also be observed in the exons of yeast and other fungi.

Generally there is an underrepresentation of ACG and ACT repeats in most taxa. The lack of ACG repeats is worth noting, because the triplet repeat with the same base composition ( $\mathrm{AGC}=\mathrm{CAG}$ ) is found much more frequently in all regions. There is also a noticeable excess of AGC repeats in exons compared to introns and intergenic regions. In primates and rodents, CCG constitutes the second most frequent repeat type in exons. CCG repeats are almost totally absent from introns. ACC repeats are relatively infrequent in intergenic regions and introns, with the exception of rodents, where their occurrence exceeds that of ATC repeats.

Apart from these general trends, a relatively unique pattern of distribution can be observed for each taxon. While intergenic CCG repeats are quite significant in all vertebrates, they are underrepresented in other taxa. In sharp contrast with this, there is a lack of CCG repeats in vertebrate introns (Tables 3 and 4). Rodents have a relatively balanced distribution of most triplet repeat types in intergenic regions and introns showing generally higher frequencies than most other taxa. AAT repeats are the most abundant in the introns of primates, vertebrates, arthropods, yeast and other fungi, whereas they come out third after AGG and AAG in rodents. Interestingly, in mammalian introns, AAC turns out to be the most frequent triplet repeat.

\section{Tetranucleotide Repeats}

Exons contain almost no tetranucleotide repeats (Tables 1 and 9). Therefore, data can only be evaluated for introns and intergenic regions. The abundance of tetranucleotide repeats in vertebrate introns and intergenic regions exceeds that of trinucleotide repeats. Repeat frequency by type shows a general dependence on the base composition of the repeat unit. Repeats with $<50 \%$ of $\mathrm{G}+\mathrm{C}$ are generally more abundant (Tables 6-8). There are, however, a few notable exceptions, e.g. AAGG, which constitutes the second most frequent tetranucleotide repeat in mammals, and the fourth one in primates and rodents. Repeats of the type AAAB, where $B$ denotes any base other than $A$, are very abundant in primates and rodents. AAAG and AAAT are also highly represented in other mammals.

\section{Pentanucleotide Repeats}

In all mammalian taxa, pentanucleotide repeats are at least as abundant as triplet repeats both in introns and intergenic regions (Table 1). They are underrepresented in exons of all taxa, whereas their frequency is comparable to that of trinucleotide repeats in introns and intergenic regions of nonmammalian genomes. In nonvertebrate taxa, they are invariably more frequent than tetranucleotide repeats. Within the whole genome, among the most common types we can always find $(\mathrm{A}+\mathrm{T})$-rich ones, such as AAAAC in primates, rodents or AAAAT in vertebrates, arthropods, C. elegans, vascular plants, and fungi as dominant tract (Tables 6-9). The exclusive dominance of AAAAB type repeats is clear for primates and a bit less striking for rodents, and occurs in vascular plants and fungi. An interesting finding is that the CpG-containing CCCCG repeat is present in the top $50 \%$ of pentanucleotide repeats found in vertebrate intergenic regions.

\section{Hexanucleotide Repeats}

Hexanucleotide repeats constitute the second most frequent type after trinucleotide repeats in exons (Table 1). In introns and intergenic regions of nonvertebrate taxa, they are generally more abundant than tetranucleotide repeats, and in C. elegans their density also exceeds that of pentanucleotide repeats.

The repeat motifs present in exons show a great variation and are relatively $(\mathrm{G}+\mathrm{C})$-rich (Table 9). A dominance of $(\mathrm{A}+\mathrm{T})$-rich repeats can be observed in primate, plant, yeast, and fungal introns and intergenic regions (Tables 7 and 8). A few telomere-like repeat motifs are also found, like AACCCT in vertebrates and fungi, or AATCCC in vertebrates and arthropods. Interestingly, AACCCT repeats are present in vertebrate introns and intergenic regions. The presence of the $(\mathrm{G}+\mathrm{C})$-rich ACCCCC motif in the top $50 \%$ of simple sequence repeats in introns of rodents and mammals is also noteworthy. Two CpG-containing repeats (AGAGCG and ACACGC) are relatively abundant in mammalian intergenic regions.

\section{Rare Repeats}

We could not find in our database subsets any of the following 27 sequence motifs in repeats longer than 12 bp: the pentanucleotide ACGCT, the hexanucleotides AAACGT, AAAGCG, AACGAG, AACGCG, AACGCT, AACGTT, AAGAGT, AAGCGC, ACACCG, ACACTG, ACCGAG, ACGACT, ACGATC, ACGCCT, ACGCGT, ACGCTC, ACGGCT, ACTAGC, AGATCT, AGCGCT, AGCTCG, ATATCG, ATCGCG, ATGCGC, CCCGGG, and CCGCGG. It should be noted here that 23 of them contain the dinucleotide $\mathrm{CpG}$ and four of them contain two CpG motifs. Ten of them are palindromes. Of the four hexanucleotides that do not contain the CpG dinucleotide (AAGAGT, ACACTG, ACTAGC, AGATCT), the first three include the trinucleotide duplex (ACT)•(AGT), and three contain a stop codon in at least one frame. Considering the cumulated size $(>380$ Mbp, see Table 10) of the sequences we analyzed, the 
Table 10. Cumulated Lengths of Sequences Analyzed

\begin{tabular}{lrrrr}
\hline $\begin{array}{l}\text { Taxonomic } \\
\text { group }\end{array}$ & $\begin{array}{c}\text { All } \\
(\mathrm{Mbp})^{\mathrm{a}}\end{array}$ & $\begin{array}{c}\text { Intergenic } \\
\text { regions } \\
(\mathrm{Mbp})^{\mathrm{a}}\end{array}$ & $\begin{array}{r}\text { Introns } \\
(\mathrm{Mbp})^{\mathrm{a}}\end{array}$ & $\begin{array}{c}\text { Exons } \\
(\mathrm{Mbp})^{\mathrm{a}}\end{array}$ \\
\hline $\begin{array}{l}\text { Primates } \\
\text { Human }\end{array}$ & 160.08 & 38.29 & 17.78 & 3.17 \\
$\quad$ chromosome 22 & 33.48 & - & - & - \\
Rodentia & 21.26 & 6.82 & 3.51 & 2.59 \\
Mammalia & 3.61 & 1.17 & 0.74 & 0.84 \\
Vertebrata & 5.47 & 1.92 & 1.32 & 1.19 \\
Arthropoda & 28.76 & 3.62 & 1.66 & 3.17 \\
C. elegans & 81.55 & 32.97 & 25.38 & 19.08 \\
Embryophyta & 48.17 & 15.34 & 6.44 & 10.76 \\
S. cerevisiae & 15.18 & 3.28 & 0.77 & 7.77 \\
Fungi & 17.78 & 5.79 & 1.07 & 9.28 \\
\hline a Mbp) megabase pair. & & &
\end{tabular}

total absence of a repeat type may well indicate either a sequence unpreferred for the mechanism generating repeats or strong selective pressure against repeated occurrence of the particular sequence. The very low frequency of ACT trinucleotide repeats in all sequences is also striking (Table 2). It cannot be explained by the presence of a stop codon on one strand since genomic regions other than exons are also affected.

\section{Repeats Longer than $24 \mathrm{bp}$}

The above results apply to repeats longer than $12 \mathrm{bp}$. To be able to estimate the instability of the various repeat motifs, we also analyzed repeats longer than 24 bp and defined the expandability of a repeat motif as the total length of repeats longer than $24 \mathrm{bp}$ divided by the total length of repeats longer than $12 \mathrm{bp}$. The overall distribution of these longer repeats follows comparable trends as presented above for all repeats considered (data not shown; for details see the SSRDB database at http://genetics.elte.hu/ssr). The contribution of SSRs with different unit lengths is generally similar to that observed for repeats longer than $12 \mathrm{bp}$, albeit with modified ratios. Mononucleotide repeats are, however, replaced by dinucleotide repeats as the dominant repeat type in primate, plant and yeast intergenic regions and introns. Although the abundance of the repeats longer than $24 \mathrm{bp}$ is much lower and some motifs are missing, the relative frequencies of various motifs are mostly conserved. An interesting exception is the AAC repeat in the exons of embryophytes, being much more abundant using the greater length threshold than AAG, which is the most frequent repeat at the shorter threshold (101bp/Mbp vs. 18bp/Mbp compared with $253 \mathrm{bp} / \mathrm{Mbp}$ vs. $317 \mathrm{bp} / \mathrm{Mbp}$ for AAC vs. AAG).

The contribution of repeats longer than $24 \mathrm{bp}$ to the observed SSR distribution is well represented by the expandability values, which not surprisingly, turn out to be repeat- and taxon-dependent. In all sequences, rodents show the highest and arthropods the lowest values (data not shown). The expandability of AC, AG, and AT repeats is almost uniformly high, although a preference for long $(\mathrm{AC})_{\mathrm{n}} \bullet(\mathrm{GT})_{\mathrm{n}}$ repeats is observed in primates. However, consistent with their general underrepresentation, no CG repeats longer than $24 \mathrm{bp}$ were found. In rodent intergenic regions and introns, AC, AG, and AT dinucleotide repeats show very high expandability values $(55 \%-80 \%)$, and most of these repeats are longer than $24 \mathrm{bp}$ in rodent exons (79\%$100 \%)$, even though dinucleotide repeats are generally rare in exons. In the case of trinucleotide repeats, repeat abundance and expandability rarely correlate: e.g., in primate intergenic regions, the second most abundant AAC displays the lowest expandability $(10 \%)$, whereas $45 \%$ of the total length of the moderately frequent AAG originates from tracts longer than 24 bp. Trinucleotide repeats in exons exhibit uniformly low expandability: AGC is the only trinucleotide motif for which repeats longer than $24 \mathrm{bp}$ can be found in all taxa. However, the expandability values for AGC in exons vary between 3\% (arthropods) and $57 \%$ (rodents).

\section{DISCUSSION}

We examined the distribution of microsatellites composed of motifs 1-6 bp long in primates, other mammals, other vertebrates, arthropods, C. elegans, embryophytes, $S$. cerevisiae and other fungi. To obtain a detailed picture, we analyzed the frequencies of perfect SSRs longer than $12 \mathrm{bp}$ in exons, introns, and intergenic regions for all of these taxa. Our results show that the abundance of certain repeat types varies with the genomic region and distribution is also characteristic of the taxonomic group examined.

It should be noted here that due to biased sequence availability in the databases, our results apply mainly to those regions of the genomes that contain protein-coding genes. Even in the case of 'all' sequences, where we did not select for genes (see Methods), the contribution of gene-rich sequences is considerable, as can be judged from the relatively high ratio of exon sequences compared to the total (Table 10). In an attempt to analyze regions less represented in GenBank, we included the human chromosome 22 sequence. Data obtained for this chromosome agree well with those obtained for all primate sequences, although an increase in $(\mathrm{A}+\mathrm{T})$-rich microsatellites could be observed. We suggest that the poly(A/T) tails of densely scattered retroposed sequences, like Alu, LINE-1, and processed pseudogenes are responsible for this higher proportion of $(\mathrm{A}+\mathrm{T})$-rich repeats. Chromosome 22 sequence, however, includes only the euchromatic portion, namely the relatively gene-rich long arm, 22q (Dunham et al. 1999). Thus, any interpreta- 
tion of the results should bear in mind that telomeric regions or genomic regions with very low gene density are not covered in the present analysis. Repeat abundance and distribution in such regions may differ from those presented here.

Nonetheless, analysis of the datasets resulted in several noteworthy findings. First, it is very interesting to compare repeat occurrence in introns and intergenic regions. Whereas the constraints shaping proteincoding DNA sequences obviously differ from those that affect these two regions of the genome, comparison of the latter could reveal some less trivial differences. In all vertebrates, the microsatellite distribution in introns and intergenic regions is quite similar but the abundance of CCG triplets differs: Introns do not contain this type of repeat whereas it is relatively abundant in intergenic regions. Because CCG is one of the most abundant repeats in vertebrate exons, a potential bias caused by error in distinguishing exons and intergenic regions cannot be ignored (see Methods). However, we have taken sufficient and appropriate measures to avoid such errors, and we argue that the observed difference is not due to incorrect assignment of exon sequences to intergenic regions. A short calculation carried out on primate data supports this argument: Assuming that microsatellite distribution in intergenic sequences is identical to that of introns, and the increased length of CCG repeats observed in the intergenic regions can be attributed only to exonic sequences, the expected total length of AGC repeats (the dominant trinucleotide repeat of all vertebrate exons) would be almost three times greater in intergenic regions than the observed value.

The absence of CCG and ACG repeats from introns of all vertebrates could be explained by the presence of the highly mutable CpG dinucleotide within the motif. The elevated level of CCG repetition could be found in intergenic regions of all vertebrates but not in the other taxonomic groups examined. This result suggests that intergenic sequences containing regulatory DNA elements are unmethylated sufficiently in all vertebrates to prevent 5-methyl-cytosine-directed spontaneous mutations that would efficiently disrupt repeated stretches of the CCG triplet, as it is observed for intronic sequences. An alternative explanation would be that a specific mechanism exists to maintain the observed level of CCG repeats in intergenic regions of all vertebrates. The role of cytosine methylation in histone deacetylation, chromatin remodeling, and gene silencing (Razin 1998) and the presence of CpG islands (Bird 1986) may account for this phenomenon. Coffee et al. (1999) demonstrated histone deacetylation as a consequence of CGG (=CCG) repeat expansion at the 5 ' end of FMR1 in fragile X-syndrome cells. Although the association with acetylated histones depends on the methylation state of DNA, we suggest that the length of the repetitive tract may be an important factor determining the level of methylation, not only in the CGG microsatellite but also in the proximal CpG island of FMR1. Boyes and Bird (1992) demonstrated that transcriptional repression by DNA methylation depends on $\mathrm{CpG}$ density. Thus, $(\mathrm{CCG})_{\mathbf{n}} \bullet(C G G)_{n}$ repeats may play an active role in vertebrates by allowing regulatory switches via the processes of DNA methylation/demethylation and, consequently, histone acetylation/deacetylation. The low level of CCG repeats in intergenic regions of species that do not methylate their DNA (C. elegans, Drosophila and yeast) suggests that, even in the absence of methyl-directed CpG suppression, CCG repeats are not favored outside the protein-coding regions. This supports the idea that either the maintenance of CCG repeats in intergenic regions of vertebrates or their suppression in most nonvertebrate sequences is an active process.

Another interesting problem is the absence of CCG from introns. In addition to the above mentioned effect of the CpG dinucleotide, CCG repeats may also be selected against because of the requirements of the splicing machinery. Repeated elements containing the motif GGG located at the $5^{\prime}$ end of human introns proved to be involved in splice site selection (SirandPugnet et al. 1995). Long CCG sequences could compete with this region in recruiting splicing machinery components resulting in inadequate splicing. Furthermore, CCG repeats, which exhibit considerable hairpin- and quadruplex-forming potential, may influence the secondary structure of the pre-mRNA molecule. If we consider the observations showing that intron selfcomplementarity (Howe and Ares 1997) and mRNA secondary structure (stem loops, Coleman and Roesser 1998; hairpins, Goguel et al. 1993) modulate the efficiency and accuracy of splicing, we can assume that the presence of repeated CCG tracts would interfere with the formation of mature mRNA.

Differences between introns and intergenic regions can also be observed in nonvertebrate taxa. Intergenic regions of arthropods and vascular plants show excess of AAC and AAG repeats, respectively, when compared to introns of the same taxon. In fungi, AAT is the most frequent trinucleotide repeat in both intergenic regions and introns, but its abundance is much higher in the latter. Other biases (e.g., C, AG, and AAG in C. elegans; AC in yeast and other fungi) also suggest that the selective forces acting on intergenic regions and introns differ from each other in a taxon-specific manner.

It is also worth noting that tetranucleotide repeats represent a higher proportion of all vertebrate genomes than triplet repeats (Table 1), in spite of the fact that exons seem to tolerate only trinucleotide and hexanucleotide repeats effectively. The observed dependence of repeat abundance on repeated unit length is 
very much biased from the expected trend of gradual decrease. SSRs with even unit length seem to be favored strongly in rodent introns and intergenic regions, and, to a lesser extent, in other vertebrates. In sharp contrast to this, penta- and hexanucleotide repeats are almost invariably more frequent than tetranucleotide repeats in all nonvertebrate taxa. This varying dependence on repeat unit length suggests fundamental differences between vertebrates and other taxa in the mechanisms of generation and fixation of simple repetitive DNA.

Although our analysis cannot measure microsatellite polymorphism per se, the maximum, average, and variance of SSR lengths may give good indication of the expected instability (data available online). As a rough estimate for this expandability, we compared the abundance of SSRs longer than $24 \mathrm{bp}$ to that of repeats longer than $12 \mathrm{bp}$. AC, AG, and AT dinucleotide repeats show a striking dominance among long SSRs in introns and intergenic regions of all taxa, except for fungi. This suggests that dinucleotide repeats other than CG are the most expandable types in higher eukaryotes, a statement well supported by the numerous dinucleotide microsatellite markers used in mapping studies.

Our study confirmed the previous results indicating that the microsatellite patterns of coding and noncoding regions in eukaryotes show divergence that can be explained on the basis of differential selection (Hancock 1995). However, where Hancock (1995) — using a different approach - found high correlation between introns and intergenic regions in Homo sapiens, C. elegans and S. cerevisiae, we observed characteristic differences between the two regions in all taxa examined. The notion of differential selection can also be invoked to explain these differences. Moreover, our results clearly demonstrate that the preferred SSR types in exons and other genomic regions are taxon-dependent. Each repeat type that was shown to be flexible in forming various nonconventional intra- or interstrand structures (Pearson and Sinden 1998; Sinden 1999) can be found in relatively high frequencies in one or more, but never in all, taxa. This observation may indicate differences in repair enzyme specificities or other divergent factors acting at the level of selection.

Our results show, in accordance with many other studies, that strand-slippage theories alone cannot explain microsatellite distribution in the genome as a whole. The inherent potential of a sequence to form alternative DNA conformations can be important for the generation of SSRs, but cannot account for the differences observed among taxa. Enzymes and other proteins involved in various aspects of DNA-processing (i.e., replication and repair) and chromatin remodeling may be responsible for the taxon-specificity of microsatellite abundance. It should be emphasized that not only does the repetitiveness of the genomes differ (Hancock 1996b), but also the preferred microsatellite types are quite different. This may indicate that SSRs play an important role in genome evolution whereas the processes responsible for SSR generation and fixation must also have undergone alteration during evolution.

\section{METHODS}

\section{DNA Sequences}

Sequences were obtained from GenBank releases 107 (for primates), 109 (for rodents, mammals, and vertebrates) and 110 (for all other taxa) (ftp://ncbi.nlm.nih.gov/genbank). The taxonomic groups examined were the following: primates, rodents, other mammals (excluding primates and rodents), other vertebrates (excluding mammals), arthropods, C. elegans, embryophytes, $S$. cerevisiae, and other fungi. The human chromosome 22 sequence superlink was obtained from the Sanger Center web site (http://www.sanger.ac.uk/HGP/ Chr22). Only genomic (chromosomal) sequences were included in our study. To decrease the effect of database bias as much as possible, we eliminated all GenBank entries defined as either tandem repeats, microsatellites, minisatellites, SSRs, telomeric or centromeric sequences. All mRNA, cDNA, and structural RNA sequences were excluded from the analysis. Standard UNIX tools (e.g., grep, awk) and Perl scripts were used to carry out the necessary filtering steps. From the remaining sequences, we selected those $\$ 250$-bp long (1000 bp in the case of primate sequences). The redundancy of sequences present in the database was minimized using the program CLEANUP (Grillo et al. 1996). We eliminated sequences that were $\$ 95 \%$ similar to and overlapped by $\$ 60 \%$ with another, longer sequence. The sizes of the database subsets used for the analysis, also broken down to intergenic regions, introns, and exons, are listed in Table 10. The taxonomic groups are rather arbitrarily defined, primarily based on sequence availability. The species contributing to $>5 \%$ of sequences in the appropriate database subset are listed in Table 11.

Although full chromosomal sequences are available for $S$. cerevisiae and C. elegans, the unconfirmed nature of the majority of sequence annotations prevented their meaningful use in our study. The potential risk of incorrectly classifying DNA fragments into exons, introns, and intergenic regions cannot be neglected even for sequences derived from the traditional GenBank database sections. Although the extent of such bias did not seem to be large, we tried to minimize it by excluding from the analysis all such entries that contained no CDS line and by a rather conservative handling of alternative splicing (either biologically relevant or due to uncertain predictions or database errors). We eliminated from our analysis all DNA fragments where exon-intron junctions of a proteincoding gene was specified in two or more different, contradictory ways. We also ignored putative intergenic regions before and after such genes. Despite our precautions, there still may be a few exon or intron sequences specified incorrectly as intergenic regions. We think, however, that the resultant bias should not affect our conclusions.

Because most of our results were obtained from sequences containing protein-coding genes, we were also interested in whether or not this caused a bias in the SSR distribution. To test this, we also carried out the analysis on the full 


\begin{tabular}{|c|c|c|c|}
\hline $\begin{array}{l}\text { Taxonomic } \\
\text { group }\end{array}$ & $\begin{array}{c}\text { Number } \\
\text { of } \\
\text { species }\end{array}$ & Major species ${ }^{a}$ & $\begin{array}{l}\text { Sequence } \\
\text { lengths } \\
(\%)\end{array}$ \\
\hline Primates & 64 & Homo sapiens & 99.43 \\
\hline Rodentia & 81 & $\begin{array}{l}\text { Mus musculus } \\
\text { Rattus norvegicus }\end{array}$ & $\begin{array}{l}73.71 \\
18.25\end{array}$ \\
\hline Mammalia & 203 & $\begin{array}{l}\text { Bos taurus } \\
\text { Sus scrofa } \\
\text { Oryctolagus cuniculus } \\
\text { Ovis aries } \\
\text { Canis familiaris }\end{array}$ & $\begin{array}{r}27.26 \\
20.72 \\
19.09 \\
10.59 \\
6.62\end{array}$ \\
\hline Vertebrata & 353 & $\begin{array}{l}\text { Gallus gallus } \\
\text { Fugu rubripes } \\
\text { Xenopus laevis }\end{array}$ & $\begin{array}{l}32.20 \\
17.76 \\
12.15\end{array}$ \\
\hline Arthropoda & 586 & $\begin{array}{l}\text { Drosophila } \\
\quad \text { melanogaster } \\
\text { Drosophila } s p .^{\mathrm{b}}\end{array}$ & $\begin{array}{r}84.27 \\
7.93\end{array}$ \\
\hline Embryophyta & 1313 & Arabidopsis thaliana & 79.18 \\
\hline Fungi & 1164 & $\begin{array}{l}\text { Schizosaccharomyces } \\
\text { pombe }\end{array}$ & 48.41 \\
\hline
\end{tabular}

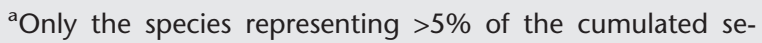
quence length are listed.

${ }^{b}$ All other Drosophila species included in the analysis (159 species).

sequence of the human chromosome 22 . The sequence was used as a whole, i.e., no attempt was made to assign portions of the chromosome 22 sequence to exon, intron, or intergenic regions.

\section{SSR Analysis}

From the database subsets obtained for each taxa, we extracted all perfect tandem repeats with a maximum unit size of six that contained at least two consecutive units, as described by Jurka and Pethiyagoda (1995). The SSRs were then grouped according to their localization in the genome (i.e., within exons, introns, or intergenic regions) using Perl scripts. This classification was based on the information provided in the CDS feature table lines of the GenBank entries. Intergenic regions were defined as being the part of DNA from the end of the last exon of one gene to the beginning of the first exon of the following gene (similar to Hancock 1995). Fragments derived from entries containing no CDS line were not classified to regions but were retained in all sequences.

Further data analysis (classification of SSRs by unit patterns and computing the values listed in the tables) was carried out as described by Jurka and Pethiyagoda (1995). In the present analysis, repeats with unit patterns being circular permutations and/or reverse complements of each other were grouped together as one type. The total number of such nonoverlapping types is 501 for $1-6$-bp long motifs (for details see Jurka and Pethiyagoda 1995).

We mainly examined the distribution of perfect repeats $>12$-bp long. Because microsatellites are often disrupted by single base substitutions, the contribution of various repetitive motifs to the overall repetitivity of the genome could be better estimated using this relatively short cutoff length. However, to assess expandability of the repeats, we also identified repeats longer than $24 \mathrm{bp}$. For a particular motif, expandability is defined as the total length of repeats longer than $24 \mathrm{bp}$ divided by the total length of repeats longer than $12 \mathrm{bp}$.

To allow direct comparisons regardless of the cumulated size of genomic regions in the database subsets, normalized total lengths of the microsatellites were calculated for $1 \mathrm{Mbp}$ of the appropriate genomic sequence type.

\section{ACKNOWLEDGMENTS}

This work was supported by grant OTKA T19278 from the Hungarian National Scientific Research Fund. We thank Ágnes Major for helpful discussion and Paul Klonowski for the computer program of tandem repeat extraction. We also thank the anonymous referees for their useful comments and suggestions.

The publication costs of this article were defrayed in part by payment of page charges. This article must therefore be hereby marked "advertisement" in accordance with 18 USC section 1734 solely to indicate this fact.

\section{REFERENCES}

Arzimanoglou, I.I., Gilbert, F., and Barber, H.R.K. 1998.

Microsatellite instability in human solid tumors. Cancer 82: $1808-1820$.

Bates, G. and Lehrach, H. 1994. Trinucleotide repeat expansions and human genetic disease. BioEssays 16: 277-284.

Beckmann, J.S. and Weber, J.L. 1992. Survey of human and rat microsatellites. Genomics 12: 627-631.

Bidichandani, S.I., Ashizawa, T., and Patel, P.I. 1998. The GAA triplet-repeat expansion in Friedreich ataxia interferes with transcription and may be associated with an unusual DNA structure. Am. J. Hum. Genet. 62: 111-121.

Bird, A.P. 1986. CpG-rich islands and the function of DNA methylation. Nature 321: 209-213.

Boyes, J. and Bird, A. 1992. Repression of genes by DNA methylation depends on $\mathrm{CpG}$ density and promoter strength: evidence for involvement of a methyl-CpG binding protein. EMBO J. 11: $327-333$.

Coffee, B., Zhang, F. Warren, S.T., and Reines, D. 1999. Acetylated histones are associated with FMR1 in normal but not fragile X-syndrome cells. Nat. Genet. 22: 98-101.

Coleman, T.P. and Roesser, J.R. 1998. RNA secondary structure: an important cis-element in rat calcitonin/CGRP pre-messenger RNA splicing. Biochemistry 37: 15941-15950.

Dunham, I., Shimizu, N., Roe, B.A., Chissoe, S., et al. 1999. The DNA sequence of human chromosome 22. Nature 402: 489-495.

Field, D. and Wills, C. 1996. Long, polymorphic microsatellites in simple organisms. Proc. R. Soc. Lond. 263: 209-215.

Gacy, A.M., Goellner, G., Juranić, N., Macura, S., and MacMurray, C.T. 1995. Trinucleotide repeats that expand in human disease form hairpin structures in vitro. Cell 81: 533-540.

Gerber, H.-P., Seipel, K., Georgiev, O., Höfferer, M., Hug, M., Rusconi, S., and Schaffner, W. 1994. Transcriptional activation modulated by homopolymeric glutamine and proline stretches. Science 263: 808-811.

Goguel, V., Wang, Y. and Rosbash, M. 1993. Short artificial hairpins sequester splicing signals and inhibit yeast pre-mRNA splicing. Mol. Cell. Biol. 13: 6841-6848.

Grillo, G., Attimonelli, M., Liuni, S., and Pesole, G. 1996. CLEANUP: A fast computer program for removing redundancies from nucleotide sequence databases. Comput. Appl. Biosci. 12: 1-8.

Hancock, J.M. 1995. The contribution of slippage-like processes to genome evolution. J. Mol. Evol. 41: 1038-1047.

. 1996a. Simple sequences in a 'minimal' genome. Nat. Genet.

14: $14-15$. 
1996b. Simple sequences and the expanding genome. BioEssays 18: 421-425.

Howe, K.J. and Ares, M., Jr. 1997. Intron self-complementarity enforces exon inclusion in a yeast pre-mRNA. Proc. Natl. Acad. Sci. USA 94: 12467-12472.

Jakupciak, J.P. and Wells, R.D. 1999. Genetic instabilities in $(\mathrm{CTG} \bullet \mathrm{CAG})$ repeats occur by recombination. J. Biol. Chem. 274: 23468-23479.

Jurka, J. and Pethiyagoda, C. 1995. Simple repetitive DNA sequences from primates: compilation and analysis. J. Mol. Evol. 40: $120-126$

Kashi, Y., King, D., and Soller, M. 1997. Simple sequence repeats as a source of quantitative genetic variation. Trends Genet. 13: 74-78.

Lagercrantz, U., Ellegren, H., and Andersson, L. 1993. The abundance of various polymorphic microsatellite motifs differs between plants and vertebrates. Nucl. Acids Res. 21: 1111-1115.

Pearson, C.E. and Sinden, R.R. 1998. Trinucleotide repeat DNA structures: Dynamic mutations from dynamic DNA. Curr. Opin. Struct. Biol. 8: 321-330.

Perutz, M.F., Johnson, T., Suzuki, M., and Finch, J.T. 1994. Glutamine repeats as polar zippers: Their possible role in inherited neurodegenerative diseases. Proc. Natl. Acad. Sci. 91: $5355-5358$.

Razin, A. 1998. CpG methylation, chromatin structure and gene silencing - a three-way connection. EMBO J. 17: 4905-4908.

Reddy, P.S. and Housman, D.E. 1997. The complex pathology of trinucleotide repeats. Curr. Opin. Cell Biol. 9: 364-372.

Richards, R.I. and Sutherland, G.R. 1992. Dynamic mutations: A new class of mutations causing human disease. Cell 70: 709-712.
1994. Simple repeat DNA is not replicated simply. Nat. Genet. 6: 114-116.

Schlötterer, C. and Tautz, D. 1992. Slippage synthesis of simple sequence DNA. Nucl. Acids Res. 20: 211-215.

Sia, E.A., Jinks-Robertson, S., and Petes, T.D. 1997. Genetic control of microsatellite instability. Mutation Research 383: 61-70.

Sinden, R.R. 1999. Trinucleotide repeats: Biological implications of the DNA structures associated with disease-causing triplet repeats. Am. J. Hum. Genet. 64: 346-353.

Sirand-Pugnet, P., Durosay, P., Brody, E., and Marie, J. 1995. An intronic (A/U)GGG repeat enhances the splicing of an alternative intron of the chicken $\beta$-tropomyosin pre-mRNA. Nucl. Acids Res. 23: 3501-3507.

Tautz, D. and Schlötterer, C. 1994. Simple sequences. Curr. Opin. Genet. Dev. 4: 832-837.

Tautz, D., Trick, M., and Dover, G. 1986. Cryptic simplicity in DNA is a major source of genetic variation. Nature 322: 652-656.

Usdin, K. 1998. NGG-triplet repeats form similar intrastrand structures: Implications for the triplet expansion diseases. Nucl. Acids Res. 26: 4078-4085.

Warren, S.T. and Nelson, D.L. 1993. Trinucleotide repeat expansions in neurological disease. Curr. Opin. Neurobiol. 3: 757-759.

Wooster, R., Cleton-Jansen, A.-M., Collins, N., Mangion, J., Cornelis, R.S., Cooper, C.S., Gusterson, B.A., Ponder, B.A.J., von Deimling, A., Wiestler, O.D. et al. 1994. Instability of short tandem repeats (microsatellites) in human cancer. Nat. Genet. 6: 152-156.

Received January 5, 2000; accepted in revised form May 4, 2000.
Genome Research 


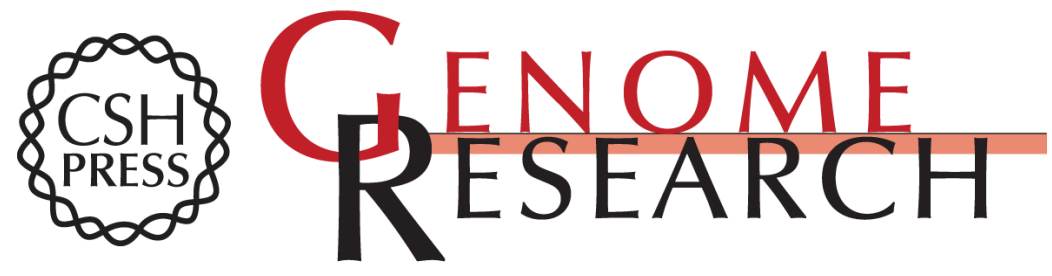

\section{Microsatellites in Different Eukaryotic Genomes: Survey and Analysis}

Gábor Tóth, Zoltán Gáspári and Jerzy Jurka

Genome Res. 2000 10: 967-981

Access the most recent version at doi:10.1101/gr.10.7.967

References This article cites 37 articles, 6 of which can be accessed free at:

http://genome.cshlp.org/content/10/7/967.full.html\#ref-list-1

\section{License}

Email Alerting Receive free email alerts when new articles cite this article - sign up in the box at the Service top right corner of the article or click here.

\section{Affordable, Accurate Sequencing.}

To subscribe to Genome Research go to: https://genome.cshlp.org/subscriptions 